\title{
Preface to special issue: LSFA 2017 and 2018
}

\author{
Sandra Alves (iD) and Renata Wassermann \\ (Received 13 November 2021; accepted 13 November 2021)
}

This special issue collects selected articles from the 12th and 13th editions of the Workshop on Logical and Semantic Frameworks, with Applications (LSFA 2017 and 2018). LSFA 2017 was held on 23-24 September 2017 in Brasília, as a satellite event of the 26th International Conference on Automated Reasoning with Analytic Tableaux and Related Methods (Tableaux 2017), the 11th International Symposium on Frontiers of Combining Systems (FroCoS 2017) and the 8th International Conference on Interactive Theorem Proving (ITP 2017). LSFA 2018 was held on 26-28 September 2018 in Fortaleza.

Logical and semantic frameworks are formal languages used to represent logics, languages and systems. These frameworks provide foundations for formal specification of systems and programming languages, supporting tool development and reasoning. LSFA aims to be a forum for presenting and discussing work in progress and therefore to provide feedback to authors on their preliminary research.

Topics of interest to this forum include, but are not limited to, automated deduction, applications of logical and/or semantic frameworks, computational and logical properties of semantic frameworks, formal semantics of languages and systems, implementation of logical and/or semantic frameworks, lambda and combinatory calculi, logical aspects of computational complexity, logical frameworks, process calculi, proof theory, semantic frameworks, specification languages and meta-languages, and type theory.

Out of the total of 29 papers that were presented at LSFA 2017 and 2018, 15 were invited to submit an extended version to this special issue. Nine manuscripts were submitted, of which four were selected for inclusion in the issue. All the submitted manuscripts were subjected to rigorous review, with some going through multiple stages of revision. The first article by Umberto Rivieccio, and Ramon Jansana on "Quasi-Nelson algebras and fragments", provides a survey on different characterisations of quasi-Nelson algebras, and in particular the characterisation as non-involutive twist algebras, as well as new results on the algebraic counterpart of the $\{\rightarrow, \sim\}$ fragment of quasi-Nelson logic. The second article by Mauricio Ayala-Rincón, Washington de Carvalho-Segundo, Maribel Fernández, Gabriel Ferreira Silva and Daniele Nantes-Sobrinho, entitled "Formalising Nominal C-Unification Generalised with Protected Variables", introduces the notion of "protected variables" in a rule-based specification of nominal C-unification formalised in Coq, which gives a generalised C-unification procedure that can be used to deal with nominal $\mathrm{C}$-matching and nominal equality check problems. The article by Amy Felty, Carlos Olarte and Bruno Xavier, entitled "A Focused Linear Logical Framework and its Application to Metatheory of Object Logics", formalises the proof of cut elimination for focused Linear Logic (LL), by proposing a set of cut rules that allow cut elimination to be proved directly on the focused system. This provides a meta-theory where the proof of cut elimination for other systems can be obtained by encoding the inference rules of other logics as LL theories and by defining the necessary conditions for those logics to have cut elimination. Finally, the article by Ernesto Copello, Nora Szasz and Alvaro Tasistro, entitled "Formalization of Metatheory of the Lambda Calculus in Constructive Type Theory Using the Barendregt Variable Convention", deals with a formalisation of the Lambda Calculus in Constructive Type Theory, which introduces principles of induction and recursion on 
terms, reproducing the use of the Barendregt Variable Convention within the setting of the Agda proof assistant.

We would like to thank all involved in this special issue. First, we would like to thank all authors for submitting their contributions and for the care and attention they took in subsequent revisions. We thank all the anonymous referees for their thorough and careful reviews and all the members of MSCS involved in processing this issue. Last but not least, we would like to express our gratitude to the MSCS editor, Pierre-Louis Curien, for his support in making this issue possible.

Guest Editors

Sandra Alves

Renata Wassermann 